\title{
AMPK: ESTRUCTURA, ROL FISIOLÓGICO, REGULACIÓN Y ACCIÓN EN ALTERACIONES METABÓLICAS
}

\section{AMPK: STRUCTURE, PHYSIOLOGICAL ROLE, REGULATION AND METABOLIC DISORDERS ACTION}

\author{
Rojas Morales Eduardo Antonio ${ }^{1,2}$;Yuing Farias Tuillang Alexander ${ }^{1,3}$; Ruiz-Tagle Idiáquez Felipe Eduardo ${ }^{1,4}$; Gonzalez \\ Fuentes Patricio Antonio ${ }^{1,3} \& *$ Zelada Varas Felipe Enrique ${ }^{1}$.
}

1-Laboratorio de Investigación en Ciencias del Ejercicio y Salud

2-Servicio de Kinesiología; Universidad Santa María

3-Docente Universidad del Mar

4-Docente Universidad Santo Tomás

\begin{abstract}
ROJAS M.E.A., YUING F.T.A., RUIZ-TAGLE I.F.E., GONZALEZ F.P.A., ZELADA V.F.E. AMPK: estructura, rol fisiológico, regulación y acción en alteraciones metabólicas. Mot. Hum., 11(2): 67-74, 2010.
\end{abstract}

\section{RESUMEN}

La AMPk es un sensor del estado energético celular. Esta proteína que se activa frente a bajos niveles de ATP o del cociente ATP/ADP, está implicada en la regulación de muchos procesos metabólicos, teniendo gran importancia en la captación de glucosa y ácidos grasos libres.

Dentro de la estructura básica de la proteína cabe destacar la presencia de tres subunidades $\alpha$, $\beta$ y dentro de las cuales cabe destacar que la subunidad $\beta$ está en contacto con depósitos de glucógeno, lo que le permite interactuar rápidamente e intervenir en el metabolismo energético.

Los principales sitios de acción son el músculo esquelético, hígado y mitocondrias, generando en el músculo una mayor translocación de los GLUT y por ende mayor captación de glucosa, a nivel hepático regula la glucogenolisis y la producción de colesterol, y a nivel mitocondrial inhibe a la Malonyl CoA que inhibe a su vez a la CPT1, y por lo tanto permite un mayor ingreso de ácidos grasos libres al interior de la mitocondria para su posterior oxidación.

Por lo expuesto anteriormente y el rol que cumple esta proteína en diversas vías metabólicas, se puede apreciar la importancia de comprender los procesos y mecanismos de acción de activación de la AMPk como herramienta clínica terapéutica.

Palabras Clave: AMPK, sensores de energía celular

\section{INTRODUCCIÓN}

La AMP - proteína kinasa activada (AMPK) es un sensor del estado de la energía celular (1). Cuando la proporción ATP/ADP cae, ya sea porque el stress inhibe la producción de ATP o porque aumenta el consumo del mismo, se activa la vía catabólica para resintetizar ATP por conversión de ADP en ATP. Si la velocidad de resíntesis es menor, o la velocidad de consumo es alta se produce un incremento en los niveles de ADP y este ADP es convertido en AMP por la Adelinato Kinasa y esto sumado a la caída del ATP activará la AMPK, la cual promueve la restauración del balance energético por estimulación del catabolismo e inhibición de los procesos que consumen ATP (1). (Figura 1)

Esta proteína ha sido indicada como el elemento clave en la regulación de muchos procesos metabólicos, teniendo importancia fundamental en la mediación de captación de glucosa y ácidos grasos, además del balance entre la utilización relativa de cada uno de estos sustratos. (2)

AMPK cumple un rol fundamental en diversos procesos metabólicos en condiciones patológicas como DM II, Obesidad y dislipidemias, además posee diferentes vías de activación entre las cuales se cuenta el ejercicio físico y la mediación de los efectos de fármacos como la metformina y los AICAR (5-amino- 4 imidazolecarboxamida ribosida) $(3,4)$.

Es por todo lo anterior que es fundamental que se otorgue una revisión seria, completa y a partir de las últimas investigaciones que se están realizando. Esto es lo que en el presente documento entregamos. 
ROJAS M.E.A., YUING F.T.A., RUIZ-TAGLE I.F.E., GONZALEZ F.P.A., ZELADA V.F.E. AMPK: estructura, rol fisiológico, regulación y acción en alteraciones metabólicas. Mot. Hum., 11(2): 67-74, 2010.

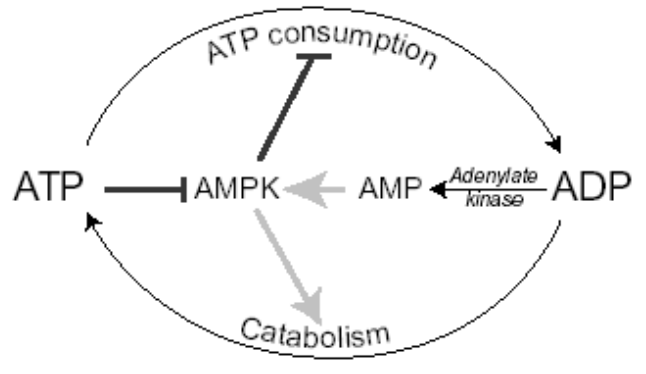

Figura 1. Rol fisiológico de AMPK en la célula. Catabolismo "cargan" la batería por la conversión de ADP a ATP, mientras que los procesos de consumo de ATP convierten el ATP en ADP. Si un stress celular causa una falla en el catabolismo o aumenta la velocidad de consumo de ATP, los niveles de ADP se elevarán y los niveles de ATP caerán. El ADP es convertido en AMP por la Adelinato Kinasa, y esto, combinado con la caída del ATP, activarán AMPK (activación alostérica). El AMPk promueve la restauración del balance energético por estimulación del catabolismo e inhibición de los procesos de consumo de ATP.

\section{Estructura del Complejo Proteína Kinasa Activada $(A M P k)$ :}

La AMPk de los mamíferos corresponde a un compuesto heterotrimétrico, es decir, está constituido de tres diferentes subunidades polipeptídicas, una subunidad catalizadora $\alpha$ y dos subunidades reguladoras $\beta$ (beta) y $\gamma$ (gamma). (1). $\mathrm{La}$ isoforma $\alpha 2$ es la subunidad encontrada predominantemente en el músculo esquelético y cardíaco, mientras que ambas subunidades $\alpha$ tienen similar distribución en el hígado (5). Entre sus dos zonas terminales posee una región autoinhibitoria, que es una secuencia proteica capaz de regular la propia actividad kinasa (2).

La subunidad $\beta$ posee una terminal para interactuar con depósitos de glicógeno, lo que le permite a la AMPk modificar rápidamente el metabolismo energético en respuesta a los cambios de la demanda metabólica (5).

\section{Regulación de la AMPk:}

La AMPk es activada por tres vías diferentes, todas ellas antagonizadas por las altas concentraciones de ATP. Las vías de activación se describen a continuación:
1. Activación alostérica por aumento en los niveles de AMP, esta vía a sido considerada la de menor grado de activación en relación a las dos vías siguientes.

2. Corresponde a una activación derivada de AMPk superiores, siendo identificados el Factor de Necrosis Tumoral (LKB 1) que fosforila a la subunidad $\alpha$. Esta vía de activación es cuantitativamente más importante que la activación alostérica (1).

3. La tercera vía corresponde a un proceso de inhibición de la desfosforilación generada en la vía anterior, por otro lado la actividad de la adelinato ciclasa que cataliza la reacción de 2 ADP hacia la formación de 1 ATP y 1 AMP es altamente sensible a los cambios del cociente ATP/AMP (1)

A nivel intramuscular, se ha sugerido que altas concentraciones de glucógeno inhibe la actividad de la AMPk, como resultado de una menor interacción entre los depósitos de glicógeno y el terminal de la subunidad $\beta$. (5)

En síntesis la activación de la AMPk depende de estímulos que llevan a la depleción de los niveles de ATP y dentro de estos estímulos se encuentra el ejercicio físico, al igual que fármacos como las Biguanidas y Thiazolidinadionas, que son utilizados en el tratamiento de la diabetes tipo II. (1)

\section{Sitios de Acción:}

La activación de la AMPk ponen en marcha las vías metabólicas para reponer los niveles de ATP, no sólo a través de la fosforilación directa, sino también a través de la regulación de transcripción de transportadores de glucosa $($ GLUT $=$ glucose transporter), genes mitocondriales en músculo e hígado (2). Es en este caso que la AMPk participa en la regulación de incorporación de glucosa en el músculo esquelético, cardíaco y adipocitos. Experimentalmente esta acción a sido inducida por ejercicio como asi también por la administración de 5 amino - 4 imidazolecarboxamida ribosita (AICAR), que genera una mayor translocación de los transportadores de glucosa. (4)

Otro destacable efecto es la inhibición que ejerce sobre la acetil CoA carboxilasa (ACC), que resulta en un bajo nivel de Malonyl CoA, el cual es un potente inhibidor de la carnitina palmitoyltransferasa 
ROJAS M.E.A., YUING F.T.A., RUIZ-TAGLE I.F.E., GONZALEZ F.P.A., ZELADA V.F.E. AMPK: estructura, rol fisiológico, regulación y acción en alteraciones metabólicas. Mot. Hum., 11(2): 67-74, 2010.

1 (CPT 1), lo que se traduce en un aumento en la incorporación de los ácidos grasos libres al interior de la mitocondria para su oxidación (5).

Ha sido comprobado que la efectividad en el tratamiento farmacológico de la diabetes mellitus tipo II (DM tipo II) mediante la administración de metformina y rosiglitazona, y el resultado de estas drogas depende del grado de activación de la AMPk, es más los cambios bioquímicos generados por estos medicamentos son similares a los generados en forma crónica por el ejercicio de sobrecarga (2).

Uno de los efectos de la activación de la AMPk se refiere a la estimulación de el óxido nítrico endotelial (eNOS) que es un potente vasodilatador e inhibe la agregación plaquetaria. Un estudio de Morrow y cols (6) comparó los efectos de activación del eNOS, mediante la activación de la AMPk entre AICAR y metformina, encontrando que la activación del eNOS por la administración de AICAR fue de 2 a 3 veces superior a la lograda con la metformina que no lo mostró cambios significativos (6).
En la figura 2 se resumen los efectos de estimulación e inhibición de la AMPK sobre los diferentes tejidos blanco.

\section{AMPk y Ejercicio Físico:}

En el músculo esquelético la AMPk puede ser activada durante la realización de ejercicio físico (7). La activación de señales periféricas en el metabolismo muscular son atribuidas como adaptaciones crónicas por la estimulación de la AMPk (8), dentro de las cuales destaca la regulación de la captación de glucosa mediante la translocación de los GLUT (Figura 3), sin embargo, estudios han evidenciado que el aumento del Calcio citosólico por contracciones musculares también media el transporte de glucosa (3.9). 
ROJAS M.E.A., YUING F.T.A., RUIZ-TAGLE I.F.E., GONZALEZ F.P.A., ZELADA V.F.E. AMPK: estructura, rol fisiológico, regulación y acción en alteraciones metabólicas. Mot. Hum., 11(2): 67-74, 2010.

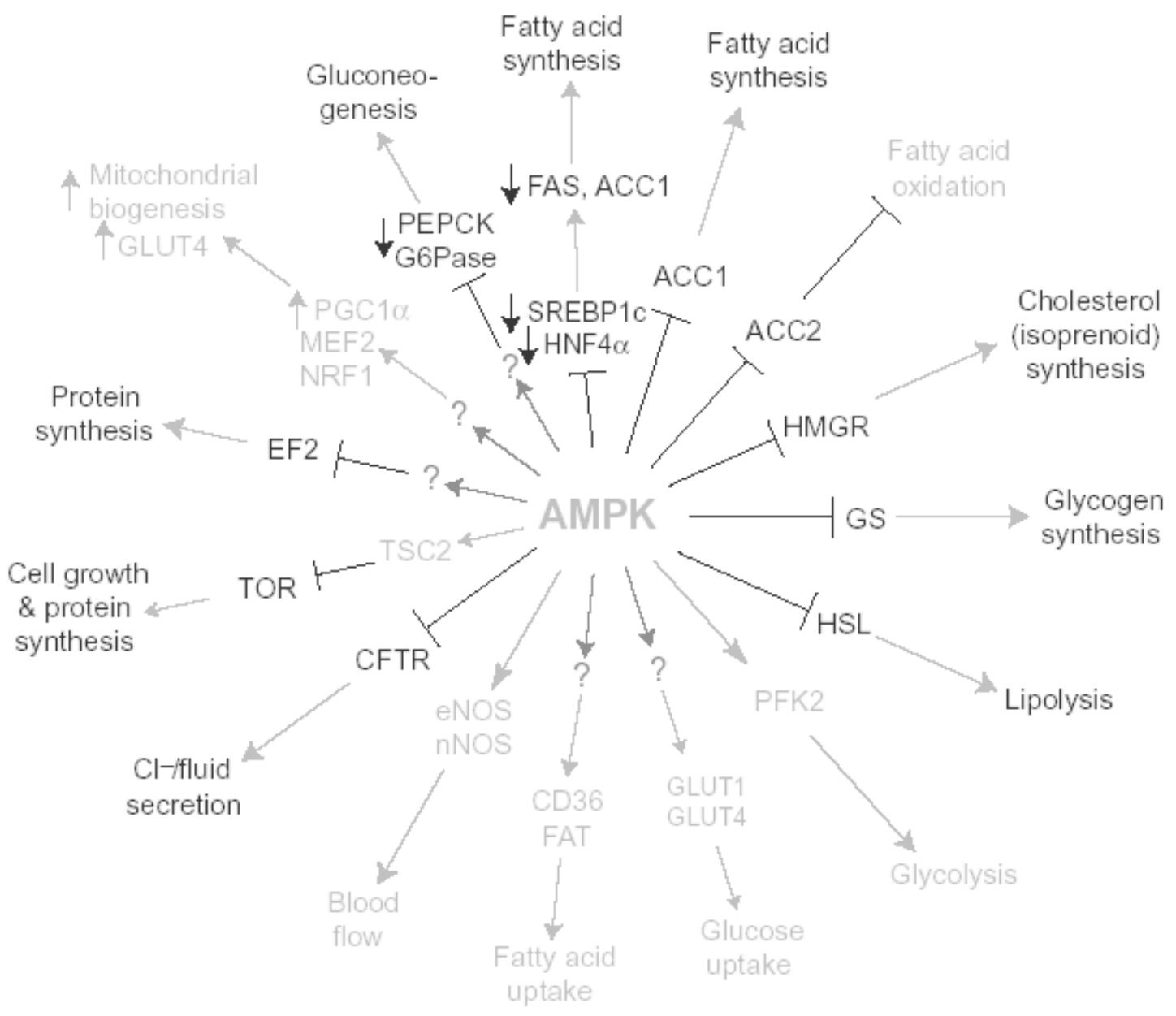

Figura 2. Tejidos Blanco de AMPK. Las proteínas blanco y los procesos activados por AMPK son mostrados en verde y los procesos que son inhibidos por AMPK en rojo. Cuando el efecto es causado por un aumento en la expresión genética, hay una flecha verde indicando el aumento, cuando hay una disminución de esa expresión genética se indica con una flecha roja. Abreviaturas. ACC1/ACC2 $(\alpha)$ y $2(\beta)$ isoformas de la Acetyl CoA Carboxilasa; CD36/FAT : CD36/ translocador de ácidos grasos; CFTR: regulador transmembrana en fibrosis quística; EF2 ; factor elongación 2; eNOS/nNOS: endotelial/neuronal isoformas de oxido nítrico; FAS ácido graso sintasa; G6Pase: glucosa 6 - fosfato; GLUT 1/4 : transportadores de glucosa; GS: glicógeno sintetasa; HMGR: 3- hidroxi-3-metil. CoA reductasa; HSL : lipasa hormona sensible; MEF2: factor específico aumentador miocito 2; NRF1 : factor respiratorio nuclear 1 ; PEPCK: fosfoenolpiruvato carboxilasa; PGC1 $\alpha$ : receptor peroxisomal proliferador activado $\gamma$ y coactivador $1 \alpha$; TOR: blanco mamífero de rapamicin. 
ROJAS M.E.A., YUING F.T.A., RUIZ-TAGLE I.F.E., GONZALEZ F.P.A., ZELADA V.F.E. AMPK: estructura, rol fisiológico, regulación y acción en alteraciones metabólicas. Mot. Hum., 11(2): 67-74, 2010.

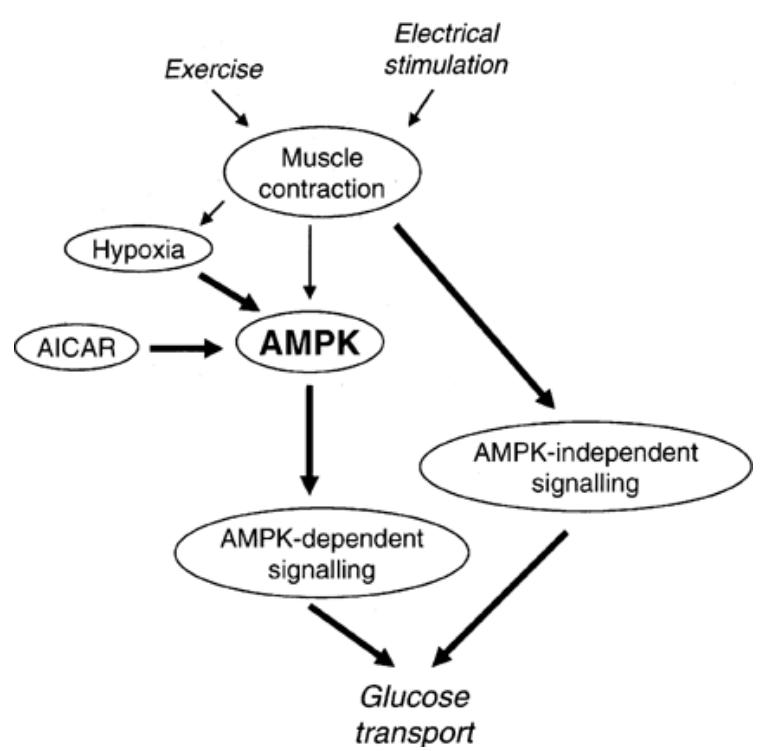

Figura 3: Modelo del rol de AMPK en la regulación del transporte de glucosa por parte del músculo esquelético en respuesta a la hipoxia, AICAR, estimulación eléctrica y ejercicio. Se propone que el aumento de captación de glucosa en respuesta la hipoxia y AICAR es dependiente de AMPK, mientras que la toma de glucosa inducida por el ejercicio no es, o bien lo es sólo en forma parcial, dependiente de AMPK.

Por otro lado es bien sabido que el entrenamiento por un largo período de tiempo puede mejorar la capacidad oxidativa del músculo mediante la biogénesis mitocondrial. Investigaciones recientes $(4,6)$, han demostrado un aumento de los niveles de enzimas oxidativas dependientes de la activación de la AMPk y por ende de la biogénesis mitocondrial, aunque los mecanismos aun no están tan claros (4).

Los estudios de sección de corte han mostrado que la activación de la AMPK y otras señales intermediarias en respuesta al ejercicio agudo está disminuido en los sujetos bien entrenados, en comparación con los no entrenados, a igual intensidad relativa de ejercicio (8). Esto sugiere que los individuos entrenados requieren un mayor estímulo para activar dichas vías (8). Se acepta, por cierto, que las adaptaciones celulares tales como el aumento de los niveles de GLUT-4 son dependientes del aumento progresivo tanto de la intensidad como el volumen (8). También se ha encontrado activación de AMPK en el ejercicio de intensidad moderada (15 a $16 \%$ de inclinación, 16 a $32 \mathrm{~m} / \mathrm{min}$, durante intervalos de 10min) (7).
Clark y cols demostraron que posterior a tres semanas de entrenamiento, realizando 7 series de $8 \mathrm{x}$ 5 minutos al $85 \%$ del VO2 max, aumentaba la actividad de AMPK $\square$ itanto antes como después del entrenamiento, pero que no aumentaba en respuesta a una única serie de ejercicio intenso (8). Por otra parte, el aumento en el contenido de la enzima AMPK $\square$ 1en sujetos entrenados sugiere que puede tener un rol importante en la adaptación al ejercicio. Langfort y colaboradores, 2003, han demostrado que tras 4 semanas de entrenamiento intenso se aumentó de manera significativa (27\%) la expresión de AMPK. Esto aumenta la posibilidad de que AMPK facilite algunas de las adaptaciones al ejercicio. (7)

La actividad de la ACC es regulada por AMPK. La fosforilación de esta ACC resulta en su desactivación y la consecuente desinhibición de la CPT1, incrementando de esta manera el potencial mitocondrial para la incorporación y oxidación de ácidos grasos. Se ha encontrado un aumento en la oxidación de grasas a ejercicios de alta intensidad (85\% VO2 max) pero no en ejercicios de moderada intensidad (65\% VO2 max). Por otra parte, se ha visto una disminución de la fosforilación de la ACC durante ejercicio prolongado de baja intensidad en el punto en que los niveles de glucógeno son bajos y aumentan los niveles de ácidos grasos libres. Esto demuestra que la persistente elevación y activación de AMPK vía fosforilación no son suficientes para mantener la fosforilación de ACC elevada hacia el final de un ejercicio prolongado (8).

La activación de la AMPk genera un aumento de la translocación de los GLUT, al igual que el aumento del Calcio citosólico, y este aumento puede ser estimulado por la ingesta de cafeína, lo que explicaría en parte, su utilización como ayuda ergogénica. Investigaciones han mostrado que los efectos sobre los niveles de calcio mediados por la administración de AICAR junto con cafeína no difieren de los niveles de contracción muscular logrados por estimulación eléctrica $(3,9)$.

\section{AMPk y Alteraciones Metabólicas:}

Es sabido que un desequilibrio metabólico es un factor patogénico temprano, común a diferentes cuadros patológicos, dentro de las cuales la principal alteración se produce a nivel del músculo esquelético 
ROJAS M.E.A., YUING F.T.A., RUIZ-TAGLE I.F.E., GONZALEZ F.P.A., ZELADA V.F.E. AMPK: estructura, rol fisiológico, regulación y acción en alteraciones metabólicas. Mot. Hum., 11(2): 67-74, 2010.

y está implicado como uno de los factores de génesis de insulino-resistencia (2). Como se ha mencionado, la AMPk corresponde a una señal intermedia del estado energético celular, además regula la inhibición de glucogenólisis, glucólisis, lipogénesis y formación de colesterol a nivel hepático (10)

Dentro de lo anterior, se ha sugerido que la activación de la AMPK en el músculo esquelético y el hígado es una excelente alternativa para el tratamiento de la diabetes tipo 2 (11). Sin embargo, existen autores que señalan que el grado de actividad de la AMPK y la expresión de isoformas son similares en los músculos de pacientes obesos, con o sin diabetes tipo 2 (10), esto indicaría que la función muscular estaría intacta en los sujetos obesos con DM tipo II, al menos en sus estadíos iniciales (10).

Finalmente, se debe señalar que la evidencia señala que la actividad de AMPK en el músculo en reposo no es regulada por las concentraciones de insulina. Por lo tanto, la falla de la insulina en activar la síntesis de glucógeno y suprimir la oxidación de lípidos en el músculo de pacientes con diabetes tipo 2 no produce cambios en la expresión o actividad de AMPK. (10) Esto reforzaría la posición de apoyo a AMPK como estrategia efectiva para tratar la obesidad y diabetes tipo 2 .

\section{CONCLUSIÓN}

Por lo expuesto en el presente trabajo, se puede apreciar que AMPK es una estructura proteica compleja que juega un rol intermediario en diversas vías metabólicas.

Si bien en los años recientes ha habido un bagaje de conocimientos cada vez más amplio acerca de AMPK, aún permanecen muchas incógnitas sobre su regulación y función, por ejemplo, refiriéndose a las señales intermedias por las cuales el ejercicio aumenta la activación de AMPK.

Existe en la actualidad un interés creciente del posible rol que juega en la expresión genética de muchos co-activadores como también su acción a nivel central en la regulación y control de los procesos de ingesta alimenticia y saciedad a través del control de la LKB1. Además de ello, también hay un gran interés en el papel de AMPK en la Diabetes tipo II, Insulina resistencia y obesidad.
Este trabajo representa una breve reseña del amplio campo de investigación de AMPK y el metabolismo energético. Se requieren a futuro mayores revisiones y enfocadas hacia aspectos más específicos de la AMPK, para así clarificar sus diversos roles y procesos involucrados en cada una de las situaciones expuestas.

\section{REFERENCIAS BIBLIOGRAFICAS}

1. Hardie, D. G. . The AMP- activated protein Kinase pathway- new players upstream and downstream. Journal of Cell Science, 2004, 117. 5479-87.

2. Kemp, B. E. , Stapleton, D. , Campbell, D. J. , Chen, Z.-P. , Murthy, S. , Walter, M. , Gupta, A. , Adams, J. J. , Katsis, F. , Van Denderen, B. , Jennings, I. G. , Iseli, T. , Michell, B. J. and Witters, L. A. . AMPK $2002-2^{\text {nd }}$ international Meeting of AMP- activated protein kinase. Biochemical Society 2003: 31; 162-68

3. Iglesias MA, Furler SM, Cooney GJ, Kraegen EW, Ye JM. AMP-activated protein kinase activation by AICAR increases both muscle fatty acid and glucose uptake in white muscle of insulin-resistant rats in vivo. Diabetes 2004: 53; 1649-54

4. Zong, H. , Ren, J. M., Young, L. H. , Pypaert, M. , Mu, J. , Birnbaum, M. J. and Shulman, G. I. . AMPK is required for mitochondrial biogenesis in skeletal muscle in response to chronic energy deprivation. PNAS, 2002, 99 (25). 15983-87

5. King, Michael W. AMPK. Medical Biochemistry 2005.http://www.indstate.edu/thcme/mwking/ho me.html

6. Morrow, V. A., Foufelle, F., Connell, J. M. C., Petrie, J. R., Gould, G. W., and Salt, I. P. . Direct Activation of AMP-activated protein kinase stimulates Nitric-oxide synthesis in Human Aortic Endothelial Cells. The Journal Of Biological Chemistry, 2003: 278 (34); 31629-39

7. Coven, D. L. , Hu, X., Lin, C., Raynald, B., Shulman, G. I. , Hardie, G. , and Young, L. H. . Physiological role of AMP-activated protein 
ROJAS M.E.A., YUING F.T.A., RUIZ-TAGLE I.F.E., GONZALEZ F.P.A., ZELADA V.F.E. AMPK: estructura, rol fisiológico, regulación y acción en alteraciones metabólicas. Mot. Hum., 11(2): 67-74, 2010.

kinase in the heart: graded activation during excercise. Am J Physiol Endocrinol Metab, 2003: $285 ; 629-36$

8. Clark, S. A.; Chen, Z.-P., Murphy, K. T., Aughey, R. J., McKenna, M. J., Kemp, B. E. and Hawley, J. A.. Intensified excercise training does not alter AMPK signaling in human skeletal muscle. Am J Physiol Endocrinol Metab, 2004: $286 ; 737-43$

9. Wright, D.C., Hucker, K. A., Holloszy, J. O. and Ho Han, D. . $\mathrm{Ca}+{ }^{2}$ and AMPK both mediate stimulation of glucose transport by muscle contractions. Diabetes, 2004: 53; 330-35

10.Højlund, K. , Mustard, K. J., Stæhr, P. , Hardie, D. G., Beck-Nielsen, H., Richter, E. A. and Wojtaszewski, J. F. P. . AMPK activity and isoform protein expression are similar in muscle of obese subjets with and without type 2 diabetes. AJP Endocrinology and metabolism, 2004: 286; $239-44$

11.Lecler, I. Rutter, G. AMP-activated kinase: A new $\square$ cell glucose sensor?. Diabetes 2004: 53 (3); 67-74 
ROJAS M.E.A., YUING F.T.A., RUIZ-TAGLE I.F.E., GONZALEZ F.P.A., ZELADA V.F.E. AMPK: estructura, rol fisiológico, regulación y acción en alteraciones metabólicas. Mot. Hum., 11(2): 67-74, 2010.

\begin{abstract}
AMPk is a sensor of cellular energy status. This protein is activated in response to low levels of ATP, or the ratio ATP/ADP, is involved in the regulation of many metabolic processes, having great importance in the uptakeof glucose and free fatty acids.

Whithin the basic structure of the protein include the presence of three subunits $\alpha, \beta y \gamma$, whithin which it is notewhorty that the $\beta$ subunit is in contact with glycogen storage, allowing you to interact and intervene quickly in the metabolism energy.

The main sitesof action are skeletal muscle, liver and mitochondria, resulting in increased muscle GLUT translocation and therefore increased uptake of glucose in the liver regulates glycogenolysis and the production of cholesterol, and inhibits mitochondrial levels of Malonyl CoA that inhibits CPT 1 to, and therefore allows a greater influx of free fatty acids into the mitochondria for further oxidation.

For the above and the role played by this protein in various metabolic pathways, one can appreciate the importance of
\end{abstract} understanding the processes and mechanisms of action of AMP $k$ as a therapeutic clinical tool.

Key words: AMPk, cellular energy sensor

Dirigir correspondencia a:

Eduardo Antonio Rojas Morales

Angamos 680. Viña del Mar, Chile

56 (032) 2578054

eduardo.rojas@usm.cl

\title{
RECIBIDO 8-11-2010
}

ACEPTADO 14-12-2010 